\title{
Prevention strategies for methicillin-resistant Staphylococcus aureus (MRSA) in Latin America
}

\begin{abstract}
After the first reports of the emergence of methicillin-resistant Staphylococcus aureus (MRSA) in the 1970s, numerous measures intended to prevent its transmission were initiated in hospitals. However, in most cases, large-scale measures failed to be implemented and the transmission of MRSA has since led to a global pandemic. Presently, doubts still remain about the best approach to prevent and control MRSA and more often than not, control measures are not implemented. Therefore, we review here the current situation in Latin America with respect to existing policies for control of MRSA, and evaluate the evidence for control measures in hospitals and the community. We look at the risk factors for infection and transmission of MRSA between hospital patients and within specific populations in the community, and at the effect of antibiotic usage on the spread of MRSA in these settings. Finally, we summarize recommendations for the prevention and control of MRSA, which can be applied to the Latin American hospital environment and community setting.
\end{abstract}

Keywords: MRSA, prevention, infection control, Latin America.

\author{
Authors \\ Carlos Alvarez ${ }^{1}$ \\ Jaime Labarca $^{2}$ \\ Mauro Salles ${ }^{3}$ \\ on behalf of the Latin \\ American Working \\ Group for Gram \\ Positive Resistance. \\ ${ }^{1}$ Hospital San Ignacio \\ and Pontificia \\ Universidad \\ Javeriana, Bogotá, \\ Colombia. \\ ${ }^{2}$ Pontificia \\ Universidad Católica \\ de Chile, Santiago, \\ Chile. \\ ${ }^{3}$ Hospital Irmandade \\ da Santa Casa de \\ Misericórdia de São \\ Paulo, São Paulo, \\ Brazil.
}

\section{INTRODUCTION}

Oxacillin-resistant Staphylococcus aureus (MRSA) is the principal micro-organism responsible for causing infections in the hospital and healthcare setting. Its prevalence has progressively increased over time and, although control measures intended to prevent its transmission were initiated in hospitals after its emergence in Europe, ${ }^{1,2}$ large-scale implementation was belated, and as a result, the transmission of MRSA has led to a global pandemic.

In addition, during more recent years, community-associated MRSA (CA-MRSA) has also increased; an incidence of 59\% of CA-MRSA in skin and soft tissue infections was reported by emergency departments in 11 US cities, ${ }^{3}$ making MRSA the most frequently isolated agent in this type of pathology. These figures, together with the risk of development of glycopeptide-resistant $S$. aureus, make the need for worldwide implementation of effective measures for the prevention of transmission of MRSA essential, both in hospitals and within the community.

Although the exact burden of MRSA-related disease remains largely unknown, consensus ex- ists among most infectious disease (ID) physicians that MRSA infections are an important clinical and public health problem. ${ }^{4,5}$ Within Latin America, although country to country variability means that data on MRSA incidence from specific hospitals may not be representative of the national situation, it is accepted that overall, the prevalence of MRSA infections in the region is probably high. ${ }^{6,7}$ However, despite published epidemiologic and microbiologic data, the best approach to prevent and control the endemic MRSA has not been agreed upon.

Hospital control of endemic MRSA has been based on several complementary strategies: early identification and isolation of asymptomatic carriers to help prevent spread; antiseptic body washes; systemic and/or topical treatment, as well as improved compliance with hand hygiene and other standard precautions to reduce carriage; and antimicrobial control measures to reduce antibiotic selection pressure. This approach may help minimize the spread of MRSA, and reduce carriage and infection rates in hospitals where MRSA is endemic. Furthermore, compliance with these measures should have a positive clini-
Correspondence to: Dr. Carlos Alvarez División de Enfermedades Infecciosas Departamento de Medicina Interna Hospital San Ignacio, and Pontificia

Universidad Javeriana Bogotá, Colombia Phone:

+57-1-3232667 or

$+57-1-5946161$

(ext 4737)

Fax: +57-1-3232667

E-mail: alvarezc@

javeriana.edu.co 
cal impact on key high-risk patients, such as those in the intensive care unit (ICU).

Published guidelines have addressed the prevention and control of MRSA transmission, including guidelines from the US Centers for Disease Control (CDC), and from the UK, Belgium, and Spain..$^{8-14}$ This article summarizes the principal recommendations to control MRSA in the hospital and community settings, and discusses their implementation in Latin America.

\section{PREVENTION AND CONTROL OF MRSA IN THE HEALTHCARE SETTING}

\section{The status of prevention and control of MRSA in hospitals in Latin America}

In 1997, the International Network for the Study and Prevention of Emerging Antimicrobial Resistance (INSPEAR) performed an observational study in hospitals in 30 countries, including six centres in Latin America (Argentina and Brazil), to help determine the prevalence and measures for control of transmission of MRSA at that time. ${ }^{15}$ Of the isolated cases of $S$. aureus, $67 \%$ were identified as MRSA, and there was an incidence of MRSA infection of 0.5 per 1,000 patient-days of hospitalization. Only $4 / 6$ centres (67\%) had an active MRSA surveillance program, with a mean proportion of one infection control nurse for every 191 hospital beds (range from 1:80 to 1:324). These rates were higher than rates in the US, European and African centers. Isolation in an individual room was used only occasionally by $50 \%$ of Latin American centers and used regularly by $0 \%$ (compared with 39.5\% and 53.5\%, respectively, in European centers). Of Latin American centers, 33\% regularly used gloves and aprons, $67 \%$ followed hand hygiene protocols and put up isolation posters on a routine basis, and 50\% had implemented an antimicrobial control program (the control methods most frequently used were limited drug lists and evaluation by an ID specialist). From this report, control of MRSA was evidently not optimal in Latin America, and since rates of MRSA infection continue to rise in many areas, implementation of control measures still needs to be improved.

\section{Risk factors for MRSA infection in hospitals}

While it is clear from case-control studies that patients infected by MRSA and patients infected or colonized by other multi-resistant bacteria share common risk factors, such as personal circumstances, length of hospital stay and use of antimicrobial agents, ${ }^{16}$ the principal risk factor for MRSA infection is the transmission of MRSA between patients. This idea is supported by the fact that $S$. aureus, or any of its antibiotic-resistant forms, is a skin-carried agent that can be easily transmitted by contact, and by the fact that dissemination in hospitals is clonal, i.e. very few clones are currently circulating throughout the world and, commonly, each hospital has only one or two clones circulating at the same time.

\section{Transmission of MRSA}

Hand-contamination of healthcare personnel represents one of the principal mechanisms for transmission of various bacterial agents from patient to patient, and has been recognized as such for more than a century. ${ }^{17}$ In numerous studies, MRSA and other multi-resistant micro-organisms, such as vancomycin-resistant enterococci (VRE), have been isolated both from the hands and gloves, aprons and other instruments used by healthcare personnel involved in the care of patients infected or colonized by these agents. ${ }^{18,19}$ Devine et al. ${ }^{20}$ reported isolation of MRSA from computer keyboards used only by doctors, while Boyce et al. (1997) ${ }^{21}$ found an incidence of $42 \%$ contamination of gloves used by nurses who had no direct contact with the patient, but who had touched surfaces in an MRSA-infected patient's room.

Contamination of healthcare personnel's clothing has also frequently been reported in the literature as a factor in the transmission of pathogens from patient to patient. Boyce et al. (1998) $)^{22}$ reported an incidence of $40 \%$ and $69 \%$ contamination of isolation and white aprons, respectively, following the care of patients colonized with MRSA or VRE. In $27 \%$ of cases, contamination was further transmitted to the hands of the healthcare personnel. In the Boyce et al. (1997) study ${ }^{21}$ MRSA was reported to contaminate aprons or uniforms worn by $65 \%$ of healthcare personnel who had cared for patients with wound or urinary infections caused by MRSA.

Instruments used in the daily care of patients, such as thermometers, sphygmomanometers, phonendoscopes and otoscopes have been reported ${ }^{23,24}$ to act as vectors for the transmission of pathogens such as MRSA and other multiresistant bacteria between patients, both through direct patient-instrument contact, and indirectly through contact with the hands of healthcare personnel. In addition, new communication technologies can easily be contaminated with MRSA and may have an impact on cross-contamination. ${ }^{25}$

Just as the equipment used in managing patients can be contaminated with MRSA, patients' rooms and the objects located in them can also act as reservoirs for the transmission of resistant agents such as MRSA. MRSA has been isolated from a wide range of objects, such as furniture, floors and cleaning utensils. ${ }^{26}$ Boyce et al. (1997) ${ }^{21}$ found that $73 \%$ of MRSA-infected and 69\% of MRSA-colonized patients' rooms had contaminated surfaces. It is important to stress that MRSA can remain on various surfaces for weeks and even months, ${ }^{27}$ thus increasing the risk of transmission. Dietze et $a l,{ }^{28}$ described cases where MRSA remained on the outer surfaces of sterile supplies for more than 38 weeks. 


\section{Use of antimicrobials}

The use of antimicrobials has been found to be a risk factor for MRSA infection, and the most consistent factor in casecontrol studies is the use of multiple antibiotics rather than one on its own (odds ratio [OR] 1.7-11.3). However, in a systematic review and meta-analysis, the risk ratio (RR) for use of single classes of antibiotics was 3 (95\% CI, 2.5-3.5) for quinolones, 2.9 (95\% CI, 2.4-3.5) for glycopeptides, 2.2 (95\% CI, 1.7-2.9) for cephalosporins and 1.9 (95\% CI, 1.72.2) for other -lactams. ${ }^{29}$

\section{EVIDENCE AND RECOMMENDATIONS FOR MRSA CONTROL MEASURES IN HOSPITALS}

\section{Standard precautions}

Standard precautions are the basis for preventing transmission of multi-resistant agents in hospital environments. However, these measures do not seem to be sufficient in the case of MRSA transmission. Jernigan et al. ${ }^{30}$ demonstrated that MRSA transmission risk was 15.6 times greater when using universal procedures than when using contact isolation in a Neonatal ICU. Marshall et al. described rates of MRSA colonization of $11.4 \%$ during hospitalization in an ICU $^{31}$ which used only universal precautions.

Recommendations for standard precautions:

- Hand hygiene is one of the most basic measures in infection control, and should be performed before and after patient examination, before entering a patient's room, and before and after wearing gloves. It should be noted that the use of gloves IS NOT a substitute for hand washing.

\section{Contact precautions}

The implementation of contact precautions is required for those patients who are colonized or infected by multi-resistant micro-organisms such as MRSA. Since clinical cultures detect only a small percentage of MRSA-colonized patients, ${ }^{32}$ and because asymptomatic colonized patients serve as reservoirs for person-to-person transmission, MRSA needs to be identified quickly through active analysis, so that contact precautions can be initiated early.

Recommendations for contact precautions:

- Contact precautions should be implemented with all patients where colonization or MRSA infection is suspected or confirmed. It involves the use of a long-sleeved isolation apron or bib, as well as gloves, which should be worn before entry into the unit, and removed before exit.

- Patients colonized or infected by MRSA should ideally stay in isolated units. If this is not possible, isolation in cohorts may be considered.

\section{Active surveillance of colonization for patients}

The use of active surveillance cultures (ASC) involves screening patients at the time of hospital admission to identify MRSA carriers (prevalent carriers), followed by periodic screening to identify patients who acquire MRSA while staying in hospital (incident cases). Numerous studies have demonstrated that the implementation of ASCs and isolation measures have made it possible to significantly reduce patient colonization and infection, while there have been reports of a gradual increase in the prevalence of MRSA in those hospitals that do not apply an active surveillance program. ${ }^{33-39}$ Calfee et al. (2002) $)^{40}$ showed a significantly lower rate of bacteremia associated with MRSA in the University of Virginia Hospital than in other hospitals that did not implement active surveillance and isolation of MRSAcolonized patients (20 cases compared with 30-70 cases of bacteremia/year in 1999).

Following hospitalization, asymptomatic MRSA carriers constitute the main reservoir and source of further spread. The Society for Healthcare Epidemiology of America (SHEA) published guidelines in 2003 supporting ASC, in combination with other basic infection control practices, to identify MRSA-colonized patients. ${ }^{11}$ The Centers for Disease Control and Prevention's Healthcare Infection Control Practices Advisory Committee offered a more conservative recommendation for ASC as a second tier for preventing transmission if baseline infection control measures fail. ${ }^{10}$ Nevertheless, questions remain regarding the viability of ASC as a worthwhile method for reducing MRSA prevalence, considering the cost associated with its implementation. Abramson et al. ${ }^{41}$ estimated that a case of MRSA bacteremia is associated with an increase in median total hospital cost of US $\$ 27,083$, while a case of methicillinsensitive $S$. aureus (MSSA) bacteremia involves an increase of US\$9,661. On the other hand, Calfee et al. (2002) (0) $^{40}$ esmated that the reduction in MRSA bacteremia as a result of active surveillance and isolation measures is associated with a saving of US\$313,596 to $\$ 975,632$ for one hospital over one year. Papia et al. ${ }^{42}$ demonstrated that early identification of six or more new MRSA-colonized patients, and the consequent decrease in transmission, makes the active surveillance program cost-effective. Karchmer et al. ${ }^{43}$ estimated that the cost of active surveillance and isolation of patients colonized and infected by MRSA was 19-27 times lower than that attributable to MRSA bacteremia as a result of an uncontrolled outbreak.

Several studies have demonstrated that ASC and isolation precautions reduce MRSA transmission. ${ }^{39,44}$ Furthermore, long-term control of MRSA is possible: Haley et al. ${ }^{45}$ reported the complete eradication of a 3-year hospital outbreak of MRSA after the implementation of ASC to identify and isolate patients colonized by MRSA, without accounting for other measures (such as restriction of the use of certain 
antibiotics). The strain responsible for this outbreak was the cause of $40 \%$ of nosocomial bacteremia cases involving MRSA and $49 \%$ of surgical site infections during this period.

In a systematic literature review, McGinigle et al. ${ }^{46}$ evaluated the association between ASC and MRSA-related events in hospitalized patients; they analyzed more than 2000 complete citations or abstracts, and found inconsistencies in the literature on ASC, which attempted to provide evidence-based conclusions. This extensive review identified only 20 of 2578 articles that met the inclusion criteria. Given the inherent difficulty and costs of performing large population-based studies, McGinigle and colleagues found few studies presenting strong scientific evidence, and none of the studies were of good quality. The authors concluded that the use of ASC reduces the incidence of MRSA infections, but the overall quality of the evidence is poor; thus, definitive, evidence-based clinical recommendations cannot be made. ${ }^{46}$ Indeed, there has been confusion regarding the precision and consistency of outcome measures in papers addressing ASC. Traditionally, ASC has been used with the primary goal of preventing MRSA transmission and a secondary long-term goal of reducing hospital-associated MRSA infection. Some studies measured the impact of ASC on MRSA control by comparing hospital-associated MRSA infection rates as the primary outcome variable, while others compared MRSA transmission rates (incident cases) as a primary outcome measure. Combining these outcome measures in the same analysis does not address the primary, clinically-significant question about whether ASCs reduce MRSA infections. Therefore, further studies are required so that more definitive recommendations can be made.

Recommendations for ASCs for patients:

- A multidisciplinary group of professionals is required to establish and develop an active surveillance program.

- The population to be subjected to this surveillance should be selected and identified on the basis of the patient's risk of being colonized by MRSA (for example: recent hospitalization, resident in an old-people's home), and the specific risk for the unit (e.g. Critical Care Unit).

- The most effective sampling technique for identifying MRSA is the nasal swab which has a negative predictive value of $98 \%,{ }^{47}$ with only $2 \%$ of patients with a negative MRSA nasal swab having a positive perianal culture. A negative nasal swab also had a lower negative predictive value for MRSA from wound cultures $(83 \%){ }^{47}$

- MRSA can be detected using culture-based methods, the most commonly-used technique. Alternatively, molecular biology techniques, the most common of which is the polymerase chain reaction (PCR), can also be used at the point of care for the screening of MRSA, with a sensitivity, specificity, positive predictive value and negative predictive value of 83.9, 98.8, 88.0 and 98.4, respectively. ${ }^{48}$ The major benefit of MRSA point-of-care testing through PCR, is the large reduction in the time to obtain a result compared with the laboratory ( $>10 \mathrm{~h}$ quicker). Furthermore, the use of PCR testing could help lower the cost of contact precaution measures.

- The surveillance program should state whether the results of the examinations should be available before contact precaution measures are implemented. Although setting these measures up before the results are obtained permits minimization of the risk of transmission from sources that have not been recognized, this is associated with greater financial costs, and therefore, each hospital will need to define its own policy according to local needs and cost restrictions.

\section{Active surveillance culture for healthcare workers}

A strong association between patient and healthcare worker (HCW) carriage was found by a recent review of MRSA outbreaks, which recommended that screening efforts should focus on HCWs with symptomatic infection. ${ }^{49}$ Albrich et al. recently published a systematic review revealing 18 studies showing proven transmission, and 26 studies showing likely transmission, to patients from MRSA-colonized HCWs. ${ }^{50}$ The latter review suggested that screening of HCWs should not be restricted to outbreak settings because there is a trend for higher colonization rates in settings where MRSA is endemic. ${ }^{50}$ Others have also suggested that screening should take place irrespective of the presence of risk factors or purulent infections, as part of pre-employment examination, or even periodically and unannounced before a work shift, to avoid detecting only transient carriers. ${ }^{49}$ However, implementing routine HCW screening is not feasible in many healthcare settings because of its high cost. Therefore, where resources are limited, priority should be given to staff working in high-risk areas, such as the ICU, dialysis areas, burn units and surgical wards (especially cardiovascular and orthopedic surgery), where prompt detection of asymptomatic carriage of MRSA by the HCW would have a greater positive impact.

An additional limitation to the systematic surveillance of HCWs is the fact that only a small proportion of HCWs have persistent colonization. ${ }^{49}$ The literature review by Albrich $e t$ al. concluded that about 5\% of HCWs become colonized with MRSA, of whom approximately 5\% develop clinical disease. Therefore, HCW most frequently act as vectors and not as the main source of MRSA transmission. ${ }^{50}$ Furthermore, HCWs may also be innocent bystanders without any role in transmission to patients, a scenario that might become increasingly frequent with rising prevalence of MRSA in the community. The dynamics of MRSA colonization in HCW are not well known and may be difficult to establish. 
Although no single approach will work universally, aggressive screening and eradication policies seem justified in outbreak investigations, or when MRSA has not reached highly endemic levels. ${ }^{50}$

Recommendations for ASC for HCWs:

- If used, screening and eradication of MRSA

colonization in HCWs should always be part of a comprehensive infection control policy, which should include staff education and emphasize high compliance with hand hygiene and contact precautions.

- Feelings of guilt or stigmatization among colonized HCWs should be avoided, and the relationship between HCWs and the infection control team should be treated with great care, avoiding any disruption.

\section{Decolonization}

The major site of staphylococcal colonization in humans is the anterior nares, although throat, perineal or gastrointestinal colonization also occurs relatively frequently. Surgical wounds, decubitus ulcers and medical device exit sites may also be colonized.

MRSA decolonization therapy can be defined as the administration of topical antimicrobial or antiseptic agents, with or without systemic antimicrobial therapy, to MRSAcolonized persons for the purpose of eradicating or suppressing the carrier state. Not all staphylococcal or MRSA carriage is the same; carriage may be transient, intermittent or persistent for months or years. The persistent carriers are more likely to be colonized at multiple sites, to transmit to others and eventually become infected. ${ }^{51}$ The main reason to consider staphylococcal decolonization is to prevent the subsequent development of infection in a colonized patient, and secondly, to prevent transmission of the organism (primarily MRSA) to others. An additional benefit of MRSA decolonization, if effective, would be to eliminate the need for use of isolation or contact precautions.

The use of MRSA decolonization therapy in conjunction with active surveillance testing may be a useful adjunctive measure for prevention of MRSA transmission within a hospital. Despite uncertainty regarding the efficacy of this approach, recommendations to consider the use of decolonization are often made based on randomized controlled trials exhibiting significant heterogeneity. Interpretation of studies addressing decolonization strategies might be confounded if transient MRSA carriers spontaneously clear their colonization, even in the absence of effective therapy. ${ }^{51}$ A recent systematic review analyzed the evidence for interventions in the prevention and control of MRSA, and showed that the current evidence does not support the routine use of topical and/or systemic antimicrobial therapy for eradicating MRSA in every colonized patient. ${ }^{52}$

Therefore, there is little evidence that decolonization reduces the risk of staphylococcal infection or transmission, and there is currently no indication for routine decolonization preoperatively, or to prevent transmission in hospitalized nonsurgical patients. Additionally, the optimal decolonization therapy regimen has not been determined. Most experience to-date has involved the use of $2 \%$ mupirocin, administered intra-nasally with or without chlorhexidine bathing. Routine washing with chlorhexidine for patients in the ICU was found to reduce the incidence of transmission of MRSA and bacteremia associated with intravascular devices. ${ }^{53}$ The use of nasal mupirocin in combination with chlorhexidine gel in a decolonization program, in association with an active surveillance program for MRSA-colonized patients, is reviewed by Calfee et al. $(2008)^{8}$ and recommendations for the decolonization regimen are proposed by Rodríguez-Baño et al. (Table 1). ${ }^{14}$ However, this is currently not possible in some Latin American countries (e.g. Brazil), where the lack of availability of nasal mupirocin prevents this type of decolonization.

Eradication of MRSA carriage in colonized HCWs or patients may be considered as a component of outbreak management in healthcare facilities. However, many studies addressing MRSA decolonization strategies among HCWs have shown marked heterogeneity in terms of the definition of successful decolonization, the length of follow-up, and the number and source of repeated specimens. ${ }^{50}$

Although S. aureus and MRSA decolonization may be achieved, at least in certain patient populations, further studies are required to identify more effective agents for long-term eradication of nasal and extra-nasal sites of colonization, to evaluate the efficacy of this strategy for preventing infection in various clinical situations, and to determine whether it has any significant role to play as an infection control measure. ${ }^{54}$ For example, adequately powered, randomized controlled trials of decolonization for preventing postoperative infections in surgical patients known to be colonized with $S$. aureus should be conducted. Outcome measures should include overall infection rates and staphylococcal infection rates. Decolonization to prevent recurrent

Table 1. Decolonization regimen for MRSAcolonized healthcare workers and patients

\begin{tabular}{|c|c|}
\hline Population & Regimen \\
\hline Health care workers & $\begin{array}{l}\text { Nasal mupirocin at } \\
2 \%+\text { washing with } \\
\text { chlorhexidine gel } 5 \mathrm{~d}\end{array}$ \\
\hline $\begin{array}{l}\text { Patients with nasal } \\
\text { colonization without skin } \\
\text { lesion/implanted devices }\end{array}$ & $\begin{array}{l}\text { Nasal mupirocin at } \\
2 \%+\text { washing with } \\
\text { chlorhexidine gel } 5 \mathrm{~d}\end{array}$ \\
\hline $\begin{array}{l}\text { Patients with skin lesions } \\
\text { or implanted devices or } \\
\text { multiple site colonization }\end{array}$ & $\begin{array}{l}\text { Nasal mupirocin at } \\
2 \%+\text { washing with } \\
\text { chlorhexidine gel } 5 \mathrm{~d} \\
\text { Systemic antibiotic } 7 \mathrm{~d}\end{array}$ \\
\hline
\end{tabular}

Adapted with permission from Rodríguez-Baño et al. (Table 4 Enferm Infecc Microbiol Clin 2008; 26(5):285-98. (C) Elsevier España). ${ }^{14}$ 
infections in patients who have CA-MRSA needs to be evaluated. ${ }^{54}$ The role of decolonization as an infection control intervention to reduce MRSA transmission needs to be defined. Ongoing surveillance of mupirocin resistance should be conducted, especially in settings with repeated or longterm mupirocin use, such as dialysis units. The effectiveness of novel agents, particularly those less likely to induce antimicrobial resistance, should be determined.

Recommendations for decolonization:

- Current evidence does not support the routine use of topical and/or systemic antimicrobial therapy for decolonization of MRSA in patients or HCWs.

- Mupirocin-susceptibility testing should be done before using this agent for decolonization.

- Decolonization with mupirocin or rifampin may be considered in those undergoing dialysis, although there is a risk of resistance emerging with long-term use of either of these drugs.

- Decolonization also may be useful in patients who have recurrent staphylococcal skin or soft tissue infection. ${ }^{14}$

\section{Environmental cleaning}

There are many studies showing control of outbreaks of MRSA with improved cleaning and/or disinfection of the environment. ${ }^{9,55,56}$ Surfaces in rooms occupied by MRSApositive patients can contaminate the hands of HCWs even in the absence of direct HCW-patient contact. ${ }^{57}$ MRSA has been isolated from various fomites, including beds, linen hampers, doorknobs and window ledges. Studies have demonstrated that these antimicrobial-resistant pathogens can persist on room surfaces even after discharge cleaning. ${ }^{58,59}$ Goodman et al. ${ }^{60}$ have recently shown that increasing the volume of disinfectant applied to environmental surfaces, providing education for environmental services staff, and instituting feedback regarding the adequacy of discharge cleaning, reduced the frequency of MRSA contamination. ${ }^{60}$ Indeed, studies have shown that environmental cleaning education and surveillance reduces levels of environmental contamination with multi-resistant pathogens.

Recommendations for environmental cleaning:

- Cleaning is essential to reduce environmental reservoirs of known hospital-associated pathogens.

- Although the goal of environmental cleaning and disinfection is not sterilization, adequate cleaning requires sufficient removal of pathogens to minimize patients' risk of acquiring infections from hospital environments. This is particularly true in areas serving high-risk patients, such as ICUs.

\section{Hospital overcrowding and understaffing}

The global epidemic of MRSA has coincided with the emergence of public health policies that promote higher patient throughput in hospitals, and has led to many services operating at near-full or full capacity. Hospital overcrowding and understaffing has resulted in the inability to adapt services to the varying number of patient admissions and available staff, ${ }^{61}$ and has led to the failure of MRSA control programs due to decreased HCW hand-hygiene compliance, increased movement of patients and staff between hospital wards, decreased levels of cohorting and overburdening of screening and isolation facilities. In turn, a high MRSA incidence increases hospital stay and bed blocking, which leads to overcrowding and a vicious cycle characterized by further failure of infection control. ${ }^{61}$

Recommendations for the control of hospital overcrowding and understaffing:

- Maintenance of an appropriate patient/staff ratio

- Epidemiologic and economic evidence

should be used in future decision-making to

evaluate the effect of health system changes

on the incidence of MRSA infection.

\section{Control of the use of antimicrobial agents}

Even though there has been a lack of strong scientific evidence supporting the strategy of antibiotic usage control for the purpose of reducing MRSA infection, different papers have already addressed this situation. Over-use of cephalosporins $s^{62-65}$ and fluoroquinolones ${ }^{66,67}$ has been associated with MRSA selection in some settings, and quinolone use has been linked with prolongation of MRSA carriage. The role of these agents for resistant of S. epidermidis is well recognized, especially with quinolones. ${ }^{68}$ SHEA guidelines lay emphasis on good antibiotic stewardship and, specifically, on fluoroquinolone control. ${ }^{11}$

Control of the use of antimicrobial agents is also a means of preventing transmission of MRSA, although the impact of this mechanism is less than that of the other control measures described above. Fukatsu et al. ${ }^{69}$ reported a reduction in rates of postoperative MRSA infection following a reduction in the use of third-generation cephalosporins in surgical prophylaxis, while Landman et al. ${ }^{70}$ demonstrated that restrictions on the use of carbapenemics, cephalosporins, vancomycin and clindamycin resulted in a reduction in the rate of infections with MRSA (from 21.9 \pm 8.1 to 17.2 \pm 7.2 patients/1000 discharges), and with ceftazidime-resistant Klebsiella pneumoniae ( $8.6 \pm 4.3$ to $5.7 \pm 4.0$ patients/1000 discharges). Likewise, Gruson et al. ${ }^{71}$ showed that a restriction on the use of ciprofloxacin and ceftazidime, along with the rotation of other -lactams, resulted in a reduction in the proportion of methicillin-resistant strains of S. aureus, causing pneumonia associated with mechanical ventilation. The proportion of resistant Gram-negative bacilli, such as Pseudomonas aeruginosa, Burkholderia cepacia, Stenotrophomonas maltophilia and Acinetobacter baumanii, was also reduced. ${ }^{71}$ In all cases, intervention in the control of antimi- 
crobials was used in conjunction with maintenance or implementation of measures to avoid patient-to-patient transmission, and therefore, interpretation of the effectiveness of this strategy is limited.

Information on the value of restriction of the use of these compounds, in particular for reducing MRSA selection, is sparse. An association between usage of mupirocin and resistance rate has been demonstrated. ${ }^{72}$ Therefore, for treatment and clearance of mupirocin-susceptible MRSA in patients with carriage or possible infection of soft tissue lesions, mupirocin should be used with a systemically active agent to improve clearance rates beyond those achieved with nasal or topical mupirocin alone. There are limited data regarding the use of oral vancomycin as prophylaxis or as part of clearance regimens for MRSA. For mupirocin-resistant strains, there are no trials to assess efficacy of other agents. A range of agents have been used topically, but the emergence of resistance to topical antibiotics is well documented. ${ }^{73}$

Recommendations for control of antimicrobials:

- While control of MRSA is based essentially on control of patient-to-patient transmission, the control of antimicrobials is a universal measure for prevention of antimicrobial resistance. The approach ensures a focus on other multi-resistant bacteria as well as MRSA. General strategies aimed at limiting the unnecessary use of antimicrobials and optimizing their efficiency should be implemented. To achieve this, it is very important that a program of antimicrobial controls be implemented in each hospital. This program should include:

- Surveillance of the consumption of antimicrobials. This is performed through co-operation with the pharmacist and is expressed as the density of consumption of antimicrobials using defined daily doses (DDD) of antibiotics, adjusted by patient days. This surveillance enables infection control committees to know whether consumption is related to trends in bacterial resistance.

- Protocols and guides for use of antimicrobials for community and hospital-associated infections and guidelines for surgical prophylaxis. Use of these protocols and guidelines constitutes an effective means for avoiding the unnecessary administration of antimicrobials and increasing the effectiveness of the prescribed regimes. The guides must be designed locally, taking resistance patterns into consideration.

- Restriction of antimicrobials. This involves the mandatory requirement to have an authorised team of professionals qualified in the use of certain antimicrobials. This selection will depend on a local decision that is based on costs and resistance patterns. Such restriction policies make it possible to reduce the use of antimicrobials and their associated cost, but with a hitherto undefined impact on bacterial resistance to antimicrobials.

- Supervision of compliance with standards and intervention programs. Implementation of all of these policies must be supervised, so as to assess adherence and in order to be able to interpret their efficacy.

\section{PREVENTION AND CONTROL OF COMMUNITY-ASSOCIATED MRSA (CA-MRSA)}

\section{The growing problem of CA-MRSA in Latin America}

CA-MRSA has been described in Latin America in Brazil, Uruguay, Colombia, Chile and Argentina. ${ }^{74-77}$ CA-MRSA has begun to replace hospital-associated isolates (HAMRSA) during recent years, especially in the USA and Taiwan, where CA-MRSA prevalence $(52 \%)$ is very high. ${ }^{78}$ The circulation and nosocomial transmission of invasive CA-MRSA infections has already been described ${ }^{79}$ in Latin American countries. This is a source of concern, as CAMRSA strains with greater virulence could be related to poorer clinical outcomes. The spread of CA-MRSA may be due to limited resources and inadequate adherence to control measures. ${ }^{80}$ This has posed a new challenge for hospitals with limited resources and their infection control committees, as endemic MRSA may be generated within a particular hospital when CA-MRSA-colonized patients are admitted without suitable screening and collection of the pertinent isolates.

The guidelines recently published by SHEA and the Infectious Diseases Society of America (IDSA) include strategies to prevent the transmission of CA-MRSA. ${ }^{8}$ In order to facilitate their implementation in the context of Latin America, we describe the adaptation of these recommendations.

\section{Risk factors and transmission of MRSA in the community}

Cases of community-acquired MRSA that have been described in Latin America have affected populations with characteristics similar to those described previously in the United States. ${ }^{74-77}$ Populations including athletes, soldiers, prisoners, homosexual men, homeless people, ethnically closed communities and children ${ }^{81-84}$ have been described with MRSA infections acquired in the community. All of these groups had no classic risk factors for MRSA transmission, i.e. diagnosis of MRSA was in the outpatient setting or within 48 hours of hospital admission, there was no medical history of MRSA infection or colonization, no medical history in the past year of hospitalization, surgery, admission to a nursing home, skilled nursing facility, hospice or dialysis unit, and no permanent indwelling catheters or medical devices that pass through the skin into the body. 
Factors that have been associated with the spread of CAMRSA skin infections include close skin-to-skin contact, openings in the skin such as cuts or abrasions, contaminated items and surfaces, crowded living conditions, poor hygiene, participation in contact sports, skin or soft tissue infection with a poor response to -lactam antibiotics, and recent or frequent antibiotic use. ${ }^{85}$

\section{Evidence for control measures in the community}

Recently, the Sociedad Española de Enfermedades Infecciosas y Microbiología Clínica (SEIMC) and Sociedad Española de Medicina Preventiva, Salud Pública e Higiene (SEMPSPH), collected the available scientific evidence on prevention and control of MRSA (including community-acquired MRSA). ${ }^{14}$ In addition, several websites of public health departments were found to have guidelines for this topic. ${ }^{80,85-87}$ Recommendations from some of these guidelines are summarized below.

\section{RECOMMENDATIONS FOR CONTROL OF MRSA IN THE COMMUNITY}

\section{Increased awareness}

Healthcare providers should be aware that community-acquired MRSA is a possibility in patients with skin and soft tissue infections and in those presenting with more severe illness compatible with $S$. aureus infection. ${ }^{80,85-87}$

\section{Early detection and appropriate treatment}

Screening methods for early signs and symptoms of skin and soft tissue infections should be implemented in correctional facilities, among contact sports participants, and in settings where persons share close living spaces (e.g. homeless shelters, camps, boarding schools, daycare settings). Close contact of persons with confirmed community-acquired MRSA infections should be monitored for signs and symptoms of MRSA infection. If treatment is necessary, healthcare providers should be informed that the patient is a close contact of an MRSA-case patient. ${ }^{87}$

\section{Reporting of community-acquired MRSA}

Outbreaks of MRSA in community settings should be reported to the local public health authorities. ${ }^{86,87}$

\section{Maintaining a clean environment}

Implementing steps to improve personal hygiene and environmental cleanliness appears to help control transmission of MRSA in crowded conditions, and where the use of shared items and equipment is common.

The following are the specific recommendations for prevention in non-hospital environments according to Los Angeles County Department of Public Health (with permission), ${ }^{86}$ which can also be used as a basis for prevention in Latin American communities.

\section{Guidelines for reducing the spread of $S$. aureus/MRSA in non-healthcare settings ${ }^{86}$}

\section{Personal hygiene}

While on the premises, patrons and staff should be encouraged to:

- Wash hands using liquid soap and water upon entering and exiting the premises and before and after any hands-on contact with other persons. Alternatively, an alcohol-based hand rub can be used according to label instructions. Visibly soiled hands should be washed with soap and water rather than an alcohol-based hand rub.

- Dry hands with disposable paper towels or air blowers (e.g. avoid sharing towels).

- Keep skin lesions (e.g. boils, insect bites, open sores or cuts) covered with a clean dry dressing.

- Limit sharing of personal items (e.g. towels, clothing and soap).

- Use a barrier (e.g. a towel or a layer of clothing) between the skin and shared equipment.

- Shower if there has been substantial skinon-skin contact with another person.

- The use of sports gloves is an option for barrier protection of the hands, provided that this is consistent with safe use of gym equipment.

\section{Shared equipment (e.g. exercise machines and massage tables)}

While using shared equipment on the premises, patrons should be encouraged to:

- Use a towel or clothing to act as a barrier between surfaces of shared equipment and bare skin.

- Wipe surfaces of equipment before and after use, especially if the surface has become wet with sweat.

- Assist facility staff with the disinfection of frequently-touched equipment surfaces if spray bottles of disinfectant are made available and instructions for use are provided.

\section{Facility staff should be encouraged to:}

- Consider making spray bottles of disinfectant available for patrons and staff to clean frequentlytouched surfaces of shared equipment between users and provide instruction (e.g. new-user orientation or posters) for the safe use of disinfectant.

- Clean shared equipment surfaces daily to remove soil.

- Disinfect shared equipment surfaces daily with a registered detergent disinfectant according to manufacturer's instructions. 
- Check with equipment manufacturers for recommendations on the appropriate maintenance of their products.

- Repair or dispose of equipment and furniture with damaged surfaces that cannot be adequately cleaned.

- Clean large surfaces (e.g. floors and tabletops) daily with a registered detergent disinfectant according to manufacturer's instructions.

- Participate in ongoing assessment and training for appropriate disinfection practices at the facility.

\section{Steam rooms and saunas}

While using these facilities, patrons should be encouraged to:

- Use a towel or clothing to act as a barrier between the benches and bare skin.

- Allow steam rooms/saunas to dry at least once a day (this will help to minimize the development of a bacterial biofilm).

- Clean and disinfect frequently touched surfaces daily.

- Consider painting wood benches with a waterproof paint, to seal and smooth the surface, facilitate drying and reduce areas where bacteria may grow.

- Ensure a halide residual (e.g. chlorine) is used as recommended for swimming pools, spa pools and other basins or tanks used for immersion by multiple patrons.

- Fill spa pools used for single-use immersion (e.g. tanks or pools that are drained after each use) with tap water and, according to manufacturer's instructions, clean the pool surfaces with a registered detergent disinfectant or with a 1:100 dilution (500-615 ppm) of household chlorine bleach.

\section{Laundry}

Staff in facility laundries should be encouraged to:

- Wash shared linens (e.g. towels, sheets, blankets or uniforms) in detergent and water at $160^{\circ} \mathrm{F}$ for at least 25 minutes, or if a lower temperature wash cycle is selected, use laundry detergent that is appropriate for cold or warm water cycles (e.g. oxygenated laundry compounds).

- Use laundry additives according to the manufacturer's instructions.

- Use a mechanical dryer on hot temperature cycle (i.e. avoid air drying).

- Distribute towels, uniforms, etc. only when they are completely dry.

\section{Recommendations for control of CA- MRSA in the healthcare setting}

According to the Spanish guidelines, the recommendations for prevention and control of CA-MRSA in the healthcare setting should be the same as for control of HA-MRSA. ${ }^{14}$

\section{PREVENTION AND CONTROL OF MRSA: CONCLUSIONS}

The effective control of MRSA is based on a group of measures, ranging from firm adherence to basic infection-control principles (such as hand hygiene), to early identification and isolation of patients colonized or infected by MRSA, as well as decolonization in specific situations. This set of measures, applied aggressively, has made it possible for countries such as Denmark and The Netherlands to have the world's lowest rates of nosocomial infections caused by MRSA. ${ }^{88,89}$ Knowledge of the risk factors, transmission mechanisms, preventive measures and local epidemiology of MRSA, will help improve compliance with the recommendations. Finally, with the increasing spread of CA-MRSA strains, it is necessary to educate not only the HCWs,${ }^{90}$ but also the general public in measures to prevent and mitigate the impact of MRSA.

\section{ACKNOWLEDGEMENTS}

\section{Financial support}

Pfizer Inc., New York, NY, USA, provided support for meetings of the Latin American Working Group on Gram Positive Resistance. Pfizer Inc. had no involvement in the collection, analysis and interpretation of data, in the writing of the manuscripts, or in the decision to submit the articles for publication.

\section{Manuscript preparation}

The support provided by Choice Pharma (Hitchin, UK), funded by Pfizer Inc., consisted of manuscript formatting and writing assistance.

\section{DISCLOSURES}

C. Alvarez: Advisory Board member for Pfizer; consultant for Pfizer, Janssen-Cilag, GlaxoSmithKline, Baxter, Merck Sharp \& Dhome and Bristol Myers Squibb.

J. Labarca: Advisory Board member for Pfizer; consultant for Pfizer, Merck Sharp \& Dhome and Wyeth; investigator for linezolid studies funded by Pfizer.

M.J.C. Salles: Advisory Board member for Pfizer and Wyeth; consultant or speaker for Pfizer, Wyeth, Merck and United Medicals.

\section{REFERENCES}

1. Barber M. Methicillin-resistant staphylococci. J Clin Pathol. 1961; 14:385-93.

2. Chabbert YA, Baudens JG. Souches de staphylocoques resistants naturellement a la meticilline et a la 5 methyl-3-phenyl4-iso-oxazyl penicilline (P12). Annales de l'Institut Pasteur. 1962; 103:222-30.

3. Moran GJ, Krishnadasan A, Gorwitz RJ et al. Methicillin-resistant $S$. aureus infections among patients in the emergency department. N Engl J Med. 2006; 355(7):666-74. 
4. Struelens MJ. Guidelines and indicators for methicillin-resistant Staphylococcus aureus control in hospitals: toward international agreement? Curr Opin Infect Dis. 2009; 22(4):337-8.

5. Cimolai N. Methicillin-resistant Staphylococcus aureus public concern, and legislative mandates. Infect Control Hosp Epidemiol. 2007; 28(7):896.

6. Guzmán-Blanco M, Mejía C, Isturiz R et al. Epidemiology of methicillin-resistant Staphylococcus aureus (MRSA) in Latin America. Int J Antimicrob Agents. 2009; 34(4):304-8.

7. Rodríguez-Noriega E, Seas C, Guzmán-Blanco M et al. Evolution of methicillin-resistant Staphylococcus aureus clones in Latin America. Int J Infect Dis. 2010; 14:e560-6.

8. Calfee DP, Salgado CD, Classen D et al. Strategies to prevent transmission of methicillin-resistant Staphylococcus aureus in acute care hospitals. Infect Control Hosp Epidemiol. 2008; 29(Suppl 1):S62-80.

9. Gould FK, Brindle R, Chadwick PR et al. Guidelines (2008) for the prophylaxis and treatment of methicillin-resistant Staphylococcus aureus (MRSA) infections in the United Kingdom. J Antimicrob Chemother. 2009; 63(5):849-61.

10. Siegel J, Rhinehart E, Jackson M, Chiarello L. Management of multidrug-resistant organisms in healthcare settings. Healthcare Infection Control Practices Advisory Committee, 2006.

11. Muto CA, Jernigan JA, Ostrowsky BE et al. SHEA guideline for preventing nosocomial transmission of multidrug-resistant strains of Staphylococcus aureus and enterococcus. Infect Control Hosp Epidemiol. 2003; 24(5):362-86.

12. Coia JE, Duckworth GJ, Edwards DI et al. Guidelines for the control and prevention of meticillin-resistant Staphylococcus aureus (MRSA) in healthcare facilities. J Hosp Infect. 2006; 63(Suppl 1):S1-44.

13. Belgian Superior Health Council. Guidelines for the control and prevention of methicillin-resistant Staphylococcus aureus transmission in Belgian hospitals. 2005.

14. Rodríguez-Baño J, Bischofberger C, Alvarez-Lerma F et al. Surveillance and control of methicillin-resistant Staphylococcus aureus in Spanish hospitals. A GEIH-SEIMC and SEMPSPH consensus document. Enferm Infecc Microbiol Clin. 2008; 26(5):285-98.

15. Richet HM, Benbachir M, Brown DE et al. Are there regional variations in the diagnosis, surveillance, and control of methicillin-resistant Staphylococcus aureus? Infect Control Hosp Epidemiol. 2003; 24(5):334-41.

16. Safdar N, Maki DG. The commonality of risk factors for nosocomial colonization and infection with antimicrobial-resistant Staphylococcus aureus, enterococcus, gram-negative bacilli, Clostridium difficile, and Candida. Ann Intern Med. 2002; 136(11):834-44.

17. Semmelweis I. The etiology, concept, and prophylaxis of childbed fever. Madison and London: University of Wisconsin Press, 1983.

18. Opal SM, Mayer KH, Stenberg MJ et al. Frequent acquisition of multiple strains of methicillin-resistant Staphylococcus aureus by healthcare workers in an endemic hospital environment. Infect Control Hosp Epidemiol. 1990; 11(9):479-85.

19. Zachary KC, Bayne PS, Morrison VJ et al. Contamination of gowns, gloves, and stethoscopes with vancomycin-resistant enterococci. Infect Control Hosp Epidemiol. 2001; 22(9):560-4.

20. Devine J, Cooke RP, Wright EP. Is methicillin-resistant Staphylococcus aureus (MRSA) contamination of ward-based computer terminals a surrogate marker for nosocomial MRSA transmission and handwashing compliance? J Hosp Infect. 2001; 48(1):72-5.
21. Boyce JM, Potter-Bynoe G, Chenevert C, King T. Environmental contamination due to methicillin-resistant Staphylococcus aureus: possible infection control implications. Infect Control Hosp Epidemiol. 1997; 18(9):622-7.

22. Boyce JM, Chenevert C. Isolation gowns prevent health care workers (HCWs) from contaminating their clothing, and possibly their hands, with methicillin-resistant Staphylococcus aureus (MRSA) and resistant enterococci. Presented at the Eighth Annual Meeting of the Society for Healthcare Epidemiology of America. Orlando, USA, 1998.

23. Brooks S, Khan A, Stoica D et al. Reduction in vancomycin-resistant Enterococcus and Clostridium difficile infections following change to tympanic thermometers. Infect Control Hosp Epidemiol. 1998; 19(5):333-6.

24. Singh D, Kaur H, Gardner WG, Treen LB. Bacterial contamination of hospital pagers. Infect Control Hosp Epidemiol. 2002; 23(5):274-6.

25. Ulger F, Esen S, Dilek A et al. Are we aware how contaminated our mobile phones with nosocomial pathogens? Ann Clin Microbiol Antimicrob. 2009; 8:7.

26. Oie S, Kamiya A. Survival of methicillin-resistant Staphylococcus aureus (MRSA) on naturally contaminated dry mops. J Hosp Infect. 1996; 34(2):145-9.

27. Neely AN, Maley MP. Survival of enterococci and staphylococci on hospital fabrics and plastic. J Clin Microbiol. 2000; 38(2):724-6.

28. Dietze B, Rath A, Wendt C, Martiny H. Survival of MRSA on sterile goods packaging. J Hosp Infect. 2001; 49(4):255-61.

29. Tacconelli E, De Angelis G, Cataldo MA et al. Does antibiotic exposure increase the risk of methicillin-resistant Staphylococcus aureus (MRSA) isolation? A systematic review and metaanalysis. J Antimicrob Chemother. 2008; 61(1):26-38.

30. Jernigan JA, Titus MG, Groschel DH et al. Effectiveness of contact isolation during a hospital outbreak of methicillin-resistant Staphylococcus aureus. Am J Epidemiol. 1996; 143(5):496-504.

31. Marshall C, Harrington G, Wolfe R et al. Acquisition of methicillin-resistant Staphylococcus aureus in a large intensive care unit. Infect Control Hosp Epidemiol. 2003; 24(5):322-6.

32. Salgado CD, Farr BM. What proportion of hospital patients colonized with methicillin-resistant Staphylococcus aureus are identified by clinical microbiological cultures? Infect Control Hosp Epidemiol. 2006; 27(2):116-21.

33. Nicolle LE, Dyck B, Thompson G et al. Regional dissemination and control of epidemic methicillin-resistant Staphylococcus aureus. Manitoba Chapter of CHICA-Canada. Infect Control Hosp Epidemiol. 1999; 20(3):202-5.

34. Kotilainen P, Routamaa M, Peltonen R et al. Eradication of methicillin-resistant Staphylococcus aureus from a health center ward and associated nursing home. Arch Intern Med. 2001; 161(6):859-63.

35. Cooper CL, Dyck B, Ormiston D et al. Bed utilization of patients with methicillin-resistant Staphylococcus aureus in a Canadian tertiary-care center. Infect Control Hosp Epidemiol. 2002; 23(9):483-4.

36. Wernitz $\mathrm{MH}$, Swidsinski S, Weist $\mathrm{K}$ et al. Effectiveness of a hospital-wide selective screening programme for methicillinresistant Staphylococcus aureus (MRSA) carriers at hospital admission to prevent hospital-acquired MRSA infections. Clin Microbiol Infect. 2005; 11(6):457-65.

37. Tomic V, Svetina Sorli P, Trinkaus D et al. Comprehensive strategy to prevent nosocomial spread of methicillin-resistant Staphylococcus aureus in a highly endemic setting. Arch Intern Med. 2004; 164(18):2038-43. 
38. Schelenz S, Tucker D, Georgeu C et al. Significant reduction of endemic MRSA acquisition and infection in cardiothoracic patients by means of an enhanced targeted infection control programme. J Hosp Infect. 2005; 60(2):104-10.

39. Lucet JC, Paoletti X, Lolom I et al. Successful long-term program for controlling methicillin-resistant Staphylococcus aureus in intensive care units. Intensive Care Med. 2005; 31(8):1051-7.

40. Calfee DP, Farr BM. Infection control and cost control in the era of managed care. Infect Control Hosp Epidemiol. 2002; 23(7):407-10.

41. Abramson MA, Sexton DJ. Nosocomial methicillin-resistant and methicillin-susceptible Staphylococcus aureus primary bacteremia: at what costs? Infect Control Hosp Epidemiol. 1999; 20(6):408-11.

42. Papia G, Louie M, Tralla A et al. Screening high-risk patients for methicillin-resistant Staphylococcus aureus on admission to the hospital: is it cost effective? Infect Control Hosp Epidemiol. 1999; 20(7):473-7.

43. Karchmer TB, Durbin LJ, Simonton BM, Farr BM. Cost-effectiveness of active surveillance cultures and contact/droplet precautions for control of methicillin-resistant Staphylococcus aureus. J Hosp Infect. 2002; 51(2):126-32.

44. Huang SS, Rifas-Shiman SL, Warren DK et al. Improving methicillin-resistant Staphylococcus aureus surveillance and reporting in intensive care units. J Infect Dis. 2007; 195(3):330-8.

45. Haley RW, Cushion NB, Tenover FC et al. Eradication of endemic methicillin-resistant Staphylococcus aureus infections from a neonatal intensive care unit. J Infect Dis. 1995; 171(3):614-24.

46. McGinigle KL, Gourlay ML, Buchanan IB. The use of active surveillance cultures in adult intensive care units to reduce methicillin-resistant Staphylococcus aureus-related morbidity, mortality, and costs: a systematic review. Clin Infect Dis. 2008; 46(11):1717-25.

47. Manian FA, Senkel D, Zack J, Meyer L. Routine screening for methicillin-resistant Staphylococcus aureus among patients newly admitted to an acute rehabilitation unit. Infect Control Hosp Epidemiol. 2002; 23(9):516-9.

48. Brenwald NP, Baker N, Oppenheim B. Feasibility study of a real-time PCR test for meticillin-resistant Staphylococcus aureus in a point of care setting. J Hosp Infect. 2009; 74(3):2459.

49. Vonberg RP, Stamm-Balderjahn S, Hansen S et al. How often do asymptomatic healthcare workers cause methicillin-resistant Staphylococcus aureus outbreaks? A systematic evaluation. Infect Control Hosp Epidemiol. 2006; 27(10):1123-7.

50. Albrich WC, Harbarth S. Health-care workers: source, vector, or victim of MRSA? Lancet Infect Dis. 2008; 8(5):289-301.

51. Bradley SF. Eradication or decolonization of methicillin-resistant Staphylococcus aureus carriage: what are we doing and why are we doing it? Clin Infect Dis. 2007; 44(2):186-9.

52. Humphreys $\mathrm{H}$. Can we do better in controlling and preventing methicillin-resistant Staphylococcus aureus (MRSA) in the intensive care unit (ICU)? Eur J Clin Microbiol Infect Dis. 2008; 27(6):409-13.

53. Bleasdale SC, Trick WE, Gonzalez IM et al. Effectiveness of chlorhexidine bathing to reduce catheter-associated bloodstream infections in medical intensive care unit patients. Arch Intern Med. 2007; 167(19):2073-9.

54. Simor AE, Daneman N. Staphylococcus aureus decolonization as a prevention strategy. Infect Dis Clin North Am. 2009; 23(1):133-51.
55. Mahamat A, MacKenzie FM, Brooker K et al. Impact of infection control interventions and antibiotic use on hospital MRSA: a multivariate interrupted time-series analysis. Int J Antimicrob Agents. 2007; 30(2):169-76.

56. Rampling A, Wiseman S, Davis L et al. Evidence that hospital hygiene is important in the control of methicillin-resistant Staphylococcus aureus. J Hosp Infect. 2001; 49(2):109-16.

57. Schmitz FJ, Verhoef J, Idel H et al. Impact of hygienic measures on the development of methicillin resistance among staphylococci between 1991 and 1996 in a university hospital. J Hosp Infect. 1998; 38(3):237-40.

58. French GL, Otter JA, Shannon KP et al. Tackling contamination of the hospital environment by methicillin-resistant Staphylococcus aureus (MRSA): a comparison between conventional terminal cleaning and hydrogen peroxide vapour decontamination. J Hosp Infect. 2004; 57(1):31-7.

59. Huang SS, Datta R, Platt R. Risk of acquiring antibiotic-resistant bacteria from prior room occupants. Arch Intern Med. 2006; 166(18):1945-51.

60. Goodman ER, Platt R, Bass R et al. Impact of an environmental cleaning intervention on the presence of methicillin-resistant Staphylococcus aureus and vancomycin-resistant enterococci on surfaces in intensive care unit rooms. Infect Control Hosp Epidemiol. 2008; 29(7):593-9.

61. Clements A, Halton K, Graves $\mathrm{N}$ et al. Overcrowding and understaffing in modern health-care systems: key determinants in meticillin-resistant Staphylococcus aureus transmission. Lancet Infect Dis. 2008; 8(7):427-34.

62. Crowcroft NS, Ronveaux O, Monnet DL, Mertens R. Methicillin-resistant Staphylococcus aureus and antimicrobial use in Belgian hospitals. Infect Control Hosp Epidemiol. 1999; 20(1):31-6.

63. Washio M, Mizoue T, Kajioka T et al. Risk factors for methicillin-resistant Staphylococcus aureus (MRSA) infection in a Japanese geriatric hospital. Public Health. 1997; 111(3):187-90.

64. Khan OA, Weston VC, Scammell BE. Methicillin-resistant Staphylococcus aureus incidence and outcome in patients with neck of femur fractures. J Hosp Infect. 2002; 51(3):185-8.

65. Muller AA, Mauny F, Bertin M et al. Relationship between spread of methicillin-resistant Staphylococcus aureus and antimicrobial use in a French university hospital. Clin Infect Dis. 2003; 36(8):971-8.

66. Harbath S, Harris AD, Carmeli Y, Samore MH. Parallel analysis of individual and aggregated data on antibiotic exposure and resistance in Gram-negative bacilli. Clin Infect Dis. 2001; 33(9):1462-8.

67. Campillo B, Dupeyron C, Richardet JP. Epidemiology of hospital-acquired infections in cirrhotic patients: effect of carriage of methicillin-resistant Staphylococcus aureus and influence of previous antibiotic therapy and norfloxacin prophylaxis. Epidemiol Infect. 2001; 127(3):443-50.

68. Hoiby N, Jarlov JO, Kemp M et al. Excretion of ciprofloxacin in sweat and multiresistant Staphylococcus epidermidis. Lancet. 1997; 349(9046):167-9.

69. Fukatsu K, Saito H, Matsuda T et al. Influences of type and duration of antimicrobial prophylaxis on an outbreak of methicillin-resistant Staphylococcus aureus and on the incidence of wound infection. Arch Surg. 1997; 132(12):1320-5.

70. Landman D, Chockalingam M, Quale JM. Reduction in the incidence of methicillin-resistant Staphylococcus aureus and ceftazidime-resistant Klebsiella pneumoniae following changes in a hospital antibiotic formulary. Clin Infect Dis. 1999; 28(5):1062-6. 
71. Gruson D, Hilbert G, Vargas F et al. Rotation and restricted use of antibiotics in a medical intensive care unit. Impact on the incidence of ventilator-associated pneumonia caused by antibiotic-resistant gram-negative bacteria. Am J Respir Crit Care Med. 2000; 162(3 Pt 1):837-43.

72. Vivoni AM, Santos KR, de-Oliveira MP et al. Mupirocin for controlling methicillin-resistant Staphylococcus aureus: lessons from a decade of use at a university hospital. Infect Control Hosp Epidemiol. 2005; 26(7):662-7.

73. Perez-Roth E, Lopez-Aguilar C, Alcoba-Florez J, Mendez-Alvarez S. High-level mupirocin resistance within methicillinresistant Staphylococcus aureus pandemic lineages. Antimicrob Agents Chemother. 2006; 50(9):3207-11.

74. Alvarez CA, Barrientes OJ, Leal AL et al. Community-associated methicillin-resistant Staphylococcus aureus, Colombia. Emerg Infect Dis. 2006; 12(12):2000-1.

75. Palombarani S, Gardella N, Tuduri A et al. Community-acquired methicillin-resistant Staphylococcus aureus infections in a hospital for acute diseases. Rev Argent Microbiol. 2007; 39(3):151-5

76. Ribeiro A, Dias C, Silva-Carvalho MC et al. First report of infection with community-acquired methicillin-resistant Staphylococcus aureus in South America. J Clin Microbiol. 2005; 43(4):1985-8.

77. Benoit SR, Estivariz C, Mogdasy C et al. Community strains of methicillin-resistant Staphylococcus aureus as potential cause of healthcare-associated infections, Uruguay, 2002-2004. Emerg Infect Dis. 2008; 14(8):1216-23.

78. Klevens RM, Morrison MA, Nadle J et al. Invasive methicillinresistant Staphylococcus aureus infections in the United States. JAMA. 2007; 298(15):1763-71.

79. Popovich KJ, Weinstein RA, Hota B. Are community-associated methicillin-resistant Staphylococcus aureus (MRSA) strains replacing traditional nosocomial MRSA strains? Clin Infect Dis. 2008; 46(6):787-94.

80. Stevens DL. Community-acquired Staphylococcus aureus infections: Increasing virulence and emerging methicillin resistance in the new millennium. Curr Opin Infect Dis. 2003; 16(3):189-91.
81. Herold BC, Immergluck LC, Maranan MC et al. Community-acquired methicillin-resistant Staphylococcus aureus in children with no identified predisposing risk. JAMA. 1998; 279(8):593-8.

82. Salgado CD, Farr BM, Calfee DP. Community-acquired methicillin-resistant Staphylococcus aureus: a meta-analysis of prevalence and risk factors. Clin Infect Dis. 2003; 36(2):131-9.

83. Farley JE. Epidemiology, clinical manifestations, and treatment options for skin and soft tissue infection caused by community-acquired methicillin-resistant Staphylococcus aureus. J Am Acad Nurse Pract. 2008; 20(2):85-92.

84. Adam H, McGeer A, Simor A. Fatal case of post-influenza, community-associated MRSA pneumonia in an Ontario teenager with subsequent familial transmission. Can Commun Dis Rep. 2007; 33(4):45-8.

85. Centers for Disease Control and Prevention. Community-associated MRSA information for clinicians. 2005.

86. Los Angeles County Department of Public Health. Guidelines for Reducing the Spread of Staph/CAMRSA in Non-Healthcare Settings v. 2. 2007.

87. California Department of Public Health Division of Communicable Disease Control. Community-associated (CAMRSA)/ Staph Infections: A Guideline for Athletic Departments. 2007.

88. European Antimicrobial Resistance Surveillance System. Annual Report. The National Institute for Public Health and Environment (RIVM), 2003.

89. Wertheim HF, Vos MC, Boelens HA et al. Low prevalence of methicillin-resistant Staphylococcus aureus (MRSA) at hospital admission in the Netherlands: the value of search and destroy and restrictive antibiotic use. J Hosp Infect. 2004; 56(4):321-5.

90. Guerra CM, Ramos MP, Penna VZ et al. Perceptions and attitudes of the professional staff concerning infection surveillance and control programs in Brazilian hospitals. Am J Infect Control. 2009; 38(1):59-62. 